\title{
Adverse effects of honey on low-density lipoprotein cholesterol and adiponectin concentrations in patients with type 2 diabetes: a randomized controlled cross-over trial
}

\section{Fatemeh Sadeghi}

Shiraz University of Medical Sciences

\section{Saedeh Salehi}

Shiraz University of Medical Sciences

Masoumeh Akhlaghi ( $\nabla$ msm.akhlaghi@gmail.com )

Shiraz University of Medical Sciences https://orcid.org/0000-0003-3868-0227

\section{Research Article}

Keywords: Honey, type 2 diabetes, LDL cholesterol, adiponectin, cross-over, trial, malondialdehyde, hs-CRP Posted Date: January 15th, 2019

DOI: https://doi.org/10.21203/rs.2.221/v1

License: (c) (1) This work is licensed under a Creative Commons Attribution 4.0 International License. Read Full License 


\section{Abstract}

Background: Due to antioxidant and fructose content, honey may be a suitable sweetener for patients with type 2 diabetes. The aim was to investigate the long-term effect of natural honey on oxidative and inflammatory status of patients with type 2 diabetes.

Methods: In a randomized controlled cross-over trial, 43 patients with type 2 diabetes were allocated to either natural honey ( $50 \mathrm{~g} /$ day) plus dietary recommendations or only dietary recommendations for 8 weeks with one month washout period in between. Dietary recommendations included weight maintenance diets for participants on honey treatment and a deficit of $-150 \mathrm{kcal}$ for those on control. Serum lipids, malondialdehyde, total antioxidant capacity, high-sensitivity C-reactive protein (hs-CRP), and adiponectin levels were measured at baseline and the end of each sequence.

Results: Malondialdehyde (10.9\%, p=0.01), hs-CRP (10.6\%, p=0.003), and adiponectin $(7.1 \%, p<0.001)$ concentrations significantly decreased in honey condition. Also, low-density lipoprotein (LDL) cholesterol $(16.8 \%, p<0.001)$ significantly decreased in control and high-density lipoprotein (HDL) cholesterol significantly increased in both conditions. Between-group differences were only statistically significant for adiponectin $(p=0.005)$ and LDL cholesterol $(p=0.005)$. Energy intake increased during honey condition $(9.4 \%, p=0.045)$ but the difference was not statistically significant between the two conditions $(p=0.1)$.

Conclusion: The results suggest that consumption of $50 \mathrm{~g} /$ day honey has adverse effects on LDL cholesterol and adiponectin levels in patients with type 2 diabetes. These adverse effects may occur because of high fructose content of honey.

Trial registration: Iranian Registry of Clinical Trials, IRCT2015062122364N2. Registered on 4 August 2015 - Retrospectively registered, available at: https://en.irct.ir/trial/19299.

Keywords: Honey, type 2 diabetes, LDL cholesterol, adiponectin, cross-over, trial, malondialdehyde, hs-CRP.

\section{Background}

Carbohydrates with a slow absorption speed are the preferred form of carbohydrates for glycemic control of patients with type 2 diabetes [1]. Due to having a facilitative transporter, fructose has the slowest intestinal absorption speed among the carbohydrates [2]. Honey is a natural sweetener with fructose constituting approximately half of its carbohydrates [3]. Thus, honey may be posed as a suitable sweetener for patients with type 2 diabetes [4]. In addition, honey can be considered as a nutritious sweetener due to possessing amino acids, minerals, vitamins, and phenolic compounds [3].

Caffeic and p-coumaric acids, quercetin, kaempferol, naringenin, chrysin, and pinocembrin are main phenolic acids and flavonoids in honey [5]. Flavonoids and phenolic compounds of honey exhibit antioxidant effects [3] which may help in alleviation of oxidative stress-associated diabetic complications [6]. Hyperglycemia and hyperlipidemia in diabetes induce oxidative stress and inflammation which lead 
to cardiovascular and other diabetic complications. Phytochemicals in honey may have suppressing effect on oxidative stress and inflammatory responses [7]. Flavonoids have also shown potential to decrease levels of blood lipids including triglycerides and low-density lipoprotein (LDL)-cholesterol [8]. By inhibition of glucose absorption, increased secretion of insulin, and decreased hepatic gluconeogenesis, polyphenols may also exert an anti-diabetic effect [9].

Despite the proposed benefits, there are health concerns for honey regarding its high fructose content [10]. Consumption of high quantities of fructose can stimulate de novo lipogenesis in the liver and increase fat deposition in the splanchnic areas including the liver itself [10]. Excessive accumulation of fat depots in the liver leads to various hepatic and metabolic derangements, such as insulin resistance and dyslipidemia, which can deteriorate control of diabetes [11].

Thus, we hypothesized that due to existence of phenolic compounds and fructose, consumption of moderate amounts of honey may have beneficial effects on oxidative stress and inflammation in patients with type 2 diabetes without adversely affecting their blood glucose. Human studies examining the effect of honey on diabetes are scarce. The few available studies have mostly focused on the effect of honey on glycemic control but no clinical trial has examined the antioxidant effect of honey on serum antioxidant capacity and markers of oxidative stress, neither in healthy individuals nor in patients with diabetes. The aim of the current study was to examine the long-term effect of natural honey on total antioxidant capacity, malondialdehyde as a marker of oxidative stress, high-sensitivity C-reactive protein (hs-CRP) and adiponectin, respectively as inflammatory and anti-inflammatory markers, in patients with type 2 diabetes.

\section{Methods}

Study design. We conducted a randomized controlled cross-over trial consisting of two 8-week interventions separated by one-month washout period on 43 patients with type 2 diabetes. The trial including recruitment and interventions was conducted in spring and summer 2015. The sample size was determined according to a previous publication [12] for detection of a mean between-group difference of $18 \mathrm{mg} / \mathrm{dl}$ for change of total cholesterol, considering a cross-over design, a statistical power of $90 \%$, and error of $5 \%$.

Subjects. Participants were 43 patients with type 2 diabetes diagnosed by an endocrinologist according to diagnostic criteria of having fasting blood glucose $\geq 126 \mathrm{mg} / \mathrm{dl}$ [13]. The participants were collected from Nader Kazemi Diabetes Clinic in Shiraz, Iran. Patients were excluded in case of malignancy, organ failure, pregnancy or lactation, smoking, fasting blood glucose $>200 \mathrm{mg} / \mathrm{dl}$, use of chemotherapy, immunosuppressive, or anti-inflammatory medications, use of hypertriglyceridemia medications, nutritional supplements, insulin therapy, change of oral hypo-glycemic and cholesterol-lowering medications, hospitalization, and affection by infectious diseases or acute medical conditions during the intervention. All participants gave written informed consent. The study was performed according to guidelines of 1964 Declaration of Helsinki and its later amendments. The trial was approved by the Ethics 
Committee of Shiraz University of Medical Sciences (approval number. CT-P-9386-8456) and registered in the Iranian Registry of Clinical Trials (IRCT 2015062122364N2).

Intervention. Participants were allocated to the sequence of either honey-control or control-honey by block randomization using a block size of 4. Two investigators enrolled the participants and an independent investigator generated the random allocation sequence and assigned the participants to the arms. The first sequence was performed for 8 weeks. Then, a washout period was run for one month, after which the second sequence of the intervention was carried out. The ethics committee of our institution did not permit having a control treatment such as glucose and sucrose because of their adverse effects on type 2 diabetes patients. The cross-over design of the study may compensate for the lack of control product because in the cross-over design each subject acts as its own control.

In honey condition, participants were recommended to consume $50 \mathrm{~g} /$ day honey plus dietary recommendations and in control condition they received only dietary recommendations. Honey was from milk vetch (Astragalus bisculatus) flowers with carbohydrate composition of $39.6 \%$ fructose, $33.3 \%$ glucose, and $3.0 \%$ sucrose as assessed by our biochemical laboratory according to the standard method [14]. The honey was natural; for which no heating process or sugar feeding for bees had been performed. Special measuring cups were provided for participants to ensure consumption of right amount of honey. The patients were asked to divide $50 \mathrm{~g}$ honey into 3 portions and consume the portions between meals with drinks like tea, coffee, or milk.

Weight maintenance diets were prescribed for all participants according to their age, sex, height, weight, body mass index, and physical activity. To ensure the suitability of weight maintenance diets a 3-week run-in period was used before the intervention. The subjects were asked to continue their diet, physical activity, and lifestyle during the study. In order to equalize energy intake between the two groups, the participants in the honey group were asked to reduce consumption of cereals by 2 servings per day. By this way, the energy deficit resulted from exclusion of 2 servings of cereals (bread or rice) was substituted with the energy obtained from consumption of honey $(150-160 \mathrm{kcal} / \mathrm{day})$. It is worth noting that the amount of the prescribed honey was within the range of recommendations for free sugar in healthy diet $[15,16]$ and according to the guidelines of the American Dietetic Association for adults with diabetes [17]. Patients were recommended to abstain from all kinds of sugar-sweetened foods and beverages. Subjects were contacted biweekly to be reminded for recommendations regarding diet and honey consumption and emphasized to keep physical activity unchanged. Also, diary sheets were provided for the subjects and they were asked to mark the sheets after each time of honey consumption.

Assessments. Dietary intakes, physical activity, anthropometric, and biochemical parameters were assessed at the beginning and the end of each sequence of the intervention. Blood was collected at baseline and the end of each sequence of the study in the morning after a 12-h overnight fast. Serum was separated by centrifugation at $3000 \mathrm{rpm}$ for $15 \mathrm{~min}$ and serum samples were frozen and kept in -70 until the end of the study. The primary outcome measure was malondialdehyde and the secondary endpoints were total antioxidant capacity and hs-CRP. 
Glucose and lipid fractions were measured by an auto-analyzer (BT 1500, Biotecnica Instruments, Italy) using specific kits (Pars Azmoon, Tehran, Iran). Concentration of malondialdehyde, a byproduct of unsaturated fatty acid peroxidation was measured, as a marker of oxidative stress, through thiobarbituric acid reactive substance (TBARS) method as described by Satoh [18]. In TBARS method, acidic condition and heat $\left(90-100^{\circ} \mathrm{C}\right)$ stimulates malondialdehyde to react with thiobarbituric acid, yielding the pink colored malondialdehyde-thiobarbituric acid adducts which can be quantified colorimetrically at $530 \mathrm{~nm}$ after extraction in organic solvents such as butanol.

Total antioxidant capacity of serum was determined using the ferric reducing capacity of plasma (FRAP) assay [19]. Briefly, $50 \mu \mathrm{l}$ of the serum was added to $1.5 \mathrm{ml}$ of freshly prepared and pre-warmed $\left(37^{\circ} \mathrm{C}\right)$ FRAP reagent ( $300 \mathrm{mM}$ acetate buffer, $\mathrm{pH}=3.6,10 \mathrm{mM}$ tripyridyl-s-triazine in $40 \mathrm{mM} \mathrm{HCl}$, and $20 \mathrm{mM}$ $\mathrm{FeCl}_{3} .6 \mathrm{H}_{2} \mathrm{O}$ in the ratio of $\left.10: 1: 1\right)$ and incubated at $37^{\circ} \mathrm{C}$ for $10 \mathrm{~min}$. The absorbance of the sample was read against reagent blank ( $1.5 \mathrm{ml}$ FRAP reagent and $50 \mu$ distilled water) at $593 \mathrm{~nm}$. Standard solutions of $\mathrm{Fe}^{2+}$ in the range of 100 to $1000 \mathrm{mM}$ were prepared from ferrous sulfate $\left(\mathrm{FeSO}_{4} \cdot 7 \mathrm{H}_{2} \mathrm{O}\right)$ in distilled water. The data was expressed as $\mathrm{mM}$ ferric ions reduced to ferrous form per $\mathrm{ml}$ (FRAP value). Levels of serum adiponectin and hs-CRP were measured by commercially available kits (IBL International, Germany) using immunoturbidimetry technique according to the manufacturer's instructions.

Trained dietitians carried out dietary assessments. Diet including all foods and drinks consumed during 3 days ( 2 random weekdays and one weekend day) was evaluated by 24 -h recall. Physical activity was assessed by self-reported validated international physical activity questionnaire and expressed as metabolic equivalent task (MET) in min/day [20]. Dietary intakes were analyzed using Nutritionist IV, version 3.5.2 (Hearst Corp., San Bruno, CA). Because Iranian food composition tables contain limited number of foods, nutrient compositions were determined using the US Department of Agriculture food composition database. For traditional Iranian foods, Iranian food composition tables were used.

Statistical analysis. Statistical analyses were performed with SPSS version 19 (SPSS Inc., Chicago, IL, USA). Data are presented as means \pm standard deviation (SD). Baseline and final values in each group were compared with paired t-test. Between-group alterations in the study outcomes were evaluated by analysis of covariance (ANCOVA) with adjustments for the corresponding basal values. Between-group comparisons of dietary intakes were tested with independent t-test. The normality of data was assessed by Kolmogorov-Smirnov test and abnormal data were log transformed where needed. $\mathrm{P}<0.05$ was considered as the significant level.

\section{Results}

Of the total of 43 subjects, 42 ( 18 males and 24 females) completed the study. One patient was excluded because of change in the dose of cholesterol-lowering medications (Figure 1). Demographic, anthropometric, and medicinal use of the participants are given in Table 1. 
There was no significant difference in fasting blood glucose within or between honey and control conditions (Table 2). LDL cholesterol significantly decreased in control condition $(P<0.001)$ and HDL cholesterol significantly increased in both honey and control conditions $(P<0.001)$. Between-condition difference was significant for $L D L$ cholesterol $(P=0.005)$. No significant change in total antioxidant capacity and concentrations of malondialdehyde, hs-CRP, and adiponectin was observed in control condition, but malondialdehyde $(P=0.01)$, hs-CRP $(P=0.003)$, and adiponectin $(P<0.001)$ concentrations significantly decreased in honey condition. Only the change in adiponectin was significantly different between the two conditions $(P=0.005)$.

Selected nutrient intakes from diet and honey are given in Table 3. Nutrient intakes of participants in the control condition did not significantly change during the intervention. In honey treatment, however, a significant increase in energy and fructose intake and an approximately significant decrease in fiber ingestion were observed. Between-group differences were only statistically significant for fructose and fiber.

\section{Discussion}

In the current study, a significant difference in adiponectin and LDL cholesterol concentrations was observed between the two conditions. These variables decreased in both conditions but participants in the honey condition showed smaller decrease in LDL cholesterol and a more evident decrease in adiponectin than those in the control, causing a statistically significant difference between the two conditions.

A limited number of clinical trials have examined the effect of honey on lipid profile of patients with diabetes, but their results are contradictory. For instance, in an 8-week clinical trial, Bahrami and colleagues [12] found a nearly significant decrease in total cholesterol, no significant change in LDL cholesterol and triglycerides, and an increase in HDL cholesterol in type 2 diabetes patients who consumed high and incremental doses of honey compared to the control. Also, Abdulrhman et al. [21] reported that consuming $0.5 \mathrm{ml} / \mathrm{kg}$ honey for 12 weeks reduced total and LDL cholesterol as well as triglycerides in patients with type 1 diabetes. Conversely, in a study by Raatz et al. [22], total and LDL cholesterol and triglycerides increased non-significantly in glucose-intolerant individuals after consuming $60 \mathrm{~g}$ honey for 2 weeks. Baseline characteristics of the subjects may explain a part of these discrepancies. Participants in the study of Raatz et al. [22] had normal levels of serum lipids whereas studies on hypercholesterolemic and hypertriglyceridemic patients have found a hypolipidemic effect of honey $[12,21]$. These results suggest that the hypolipidemic effect of honey may only be exerted in hyperlipidemic conditions. The average cholesterol and triglyceride levels in participants of this study were within the normal range; they were generally normotriglyceridemic and taking statins for their hypercholesterolemia disorder. The floral source of honey may also be the cause of contradiction between results of different studies. Phenolic compounds possess triglyceride- and cholesterol-lowering capacity [23]. A broad range of phenolic compounds has been identified in honey [3] but honeys from 
various floral sources have different phenolic constituents [4, 24, 25]. Hence, honeys from different floral origin appear different potency in lowering blood lipids.

Unexpectedly, results of this study showed that, compared to control, honey seemed to have an increasing effect on LDL cholesterol. Such effect on LDL cholesterol has been demonstrated in studies with fructose $[26,27]$. Consuming high amounts of fructose stimulates hepatic de novo lipogenesis and increases production of very low-density lipoprotein (VLDL) particles [28], which can then be converted to LDL particles in the circulation [29]. On the other hand, overproduction of VLDL and hypertriglyceridemia predisposes the individual to visceral obesity [28]. Following visceral obesity, circulating free fatty acids and hepatic triglyceride accumulation result in hepatic insulin resistance [28]. Since insulin regulates cholesterol and fatty acid synthesis in the liver, hepatic insulin resistance results in deregulation of cholesterol synthesis [30]. Silbernagel and colleagues [31] showed that high-fructose diets increase hepatic cholesterol synthesis [31]. Similarly, Stanhope et al. [32] reported that consumption of fructosesweetened, but not glucose-sweetened, beverages increases visceral adiposity, insulin resistance, and fasting concentrations of apolipoprotein B, LDL, small dense LDL, and oxidized LDL [32]. Although many of the aforementioned studies have observed the lipogenesis effect following application of high doses of fructose in healthy subjects, patients with type 2 diabetes are likely to be at a higher risk because they have degrees of visceral obesity and insulin resistance. For diabetes patients, lower doses of fructose, like what was used in this study, may produce the same adverse effects as higher doses of fructose in healthy subjects. In addition to higher fructose intake, lower fiber intake may also have contributed to higher LDL cholesterol in honey condition.

We observed a significant decrease $(7.1 \%, p<0.001)$ in serum adiponectin over the honey treatment while such magnitude of reduction was not observed in control condition $(0.07 \%, p=0.8)$. Adiponectin is an adipokine with anti-inflammatory, anti-atherogenic, and insulin sensitivity properties [33]. The effect of honey on serum adiponectin concentrations has not been examined in clinical trials but in an animal study consumption of honey ( $20 \%$ of diet weight) for one month non-significantly reduced serum adiponectin levels compared to sucrose [34]. Similar to what was mentioned for LDL cholesterol, the adiponectin-lowering effect of honey may result from its high fructose content. Since high fructose consumption promotes visceral adiposity [28] and because visceral adiposity is known to be inversely associated with circulating adiponectin concentrations [35] the lower adiponectin level in honey condition is likely due to high fructose intake. In this regard, several human studies have shown the inverse association between fructose ingestion and adiponectin levels. In one study, consumption of fructosesweetened beverages by overweight and obese adults for 10 weeks decreased adiponectin levels [36]. Also, two cross-sectional studies on adolescents demonstrated that fructose intake was inversely associated with serum adiponectin [37, 38].

In line with our hypothesis for the antioxidant effect of honey, we observed a significant reduction in serum malondialdehyde and hs-CRP during the honey treatment and a nearly significant decrease in serum total antioxidant capacity during the control condition. Although these alterations were not statistically significant between the two conditions, they suggest an antioxidant effect for the honey used 
in this study. The antioxidant effect of honey has not been examined in previous clinical trials, but a few studies investigated the effect of honey on serum CRP in hypercholesterolemic [39], glucose-intolerant [22], and overweight and obese patients [40] and reported conflicting results.

The adverse effects of honey on LDL cholesterol and adiponectin may be partly due to the addition of honey to the habitual diet. In order to avoid elevation of energy intake, the patients in the honey phase were recommended to decrease consumption of cereals by 2 servings a day. However, the analysis of dietary recalls showed that energy increased in honey condition, indicating that most of the participants did not follow diet recommendations. It is possible that the habitual intake of food has hampered the participants to decrease their energy intake. Whether substitution of honey for a part of energy intake produces promising results needs to be examined in future investigations. More extensive education and effort may be needed to ensure an appropriate adherence of the patients to dietary recommendations.

Our study had strengths and limitations. The cross-over design of the study allowed having the same patients in both conditions; so each person acted as its own control. But because the subjects were patients with type 2 diabetes, we were not permitted by the ethics committee of our institution to have a control treatment for honey. Also, as stated above, the participants did not reduce food intake during the honey condition. The results could vary if honey was substituted for a part of the habitual energy intake. This was a single-centered study, so the generalizability of the study findings is limited.

\section{Conclusion}

In conclusion, daily consumption of $50 \mathrm{~g}$ honey may have adverse effects on serum LDL cholesterol and adiponectin levels in patients with type 2 diabetes. These adverse effects are likely due to high fructose content of honey, but concomitant increased energy intake may also have a contribution.

\section{Abbreviations}

ANCOVA: analysis of covariance; BMI: body mass index; FRAP: ferric reducing capacity of plasma; HDL: high-density lipoprotein; hs-CRP: high-sensitivity C-reactive protein; LDL: low-density lipoprotein; MET: metabolic equivalent task; SD: standard deviation; TBARS: thiobarbituric acid reactive substance.

\section{Declarations}

\section{Acknowledgements}

Not applicable.

\section{Availability of data and materials}


The datasets generated during the current study are available from the corresponding author on reasonable request.

\section{Authors' contributions}

FS and MA contributed to the conception and design of the study. FS and SS recruited the participants, conducted the trial, and collected the data. FS and MA analyzed the data. FS and SS drafted the manuscript and MA revised it. All authors read and approved the final version of the manuscript.

\section{Authors' information}

FS and SS: School of Nutrition and Food Sciences, Shiraz University of Medical Sciences, Shiraz, Iran MA: Nutrition Research Center, Shiraz University of Medical Sciences, Shiraz, Iran

\section{Ethics approval and consent to participate}

All participants gave written informed consent. The study was performed according to guidelines of 1964 Declaration of Helsinki and its later amendments. The trial was approved by the Ethics Committee of Shiraz University of Medical Sciences (approval number: CT-P-9386-8456) and registered in the Iranian Registry of Clinical Trials (IRCT 2015062122364N2).

\section{Consent for publication}

Not applicable.

\section{Competing interests}

The authors declare no conflict of interest.

\section{References}

1. American Diabetes Association (2013) American Diabetes Association Releases New Nutritional Guidelines. Updated Oct 2013. Available online at: http://www.diabetes.org/newsroom/press- 
releases/2013/american-diabetes-association-releases-nutritional-

guidelines.html\#sthash.Kz08ljx4.dpuf

2. Patel C, Douard V, Yu S, et al. (2015) Transport, metabolism, and endosomal trafficking-dependent regulation of intestinal fructose absorption. FASEB J 29, 4046-4058.

3. Alvarez-Suarez JM, Giampieri F \& Battino M (2013) Honey as a source of dietary antioxidants: structures, bioavailability and evidence of protective effects against human chronic diseases. Curr Med Chem 20, 621-638.

4. Erejuwa OO (2012) The Use of Honey in Diabetes Mellitus: Is It Beneficial or Detrimental? Int J Endocrinol Metab 10, 444-445.

5. Istasse T, Jacquet N, Berchem T, Haubruge E, Nguyen BK, Richel A. Extraction of Honey Polyphenols: Method Development and Evidence of Cis Isomerization. Anal Chem Insights. 2016 Aug 10;11:49-57. doi: $10.4137 /$ ACl.S39739.

6. Ndisang JF, Vannacci A \& Rastogi S (2014) Oxidative stress and inflammation in obesity, diabetes, hypertension, and related cardiometabolic complications. Oxid Med Cell Longev 2014, 506948.

7. Akhlaghi M. Non-alcoholic Fatty Liver Disease: Beneficial Effects of Flavonoids. Phytother Res.2016 Oct;30(10):1559-1571. doi: 10.1002/ptr.5667.

8. Leyva-Soto A, Chavez-Santoscoy RA, Lara-Jacobo LR, Chavez-Santoscoy AV, Gonzalez-Cobian LN. Daily Consumption of Chocolate Rich in Flavonoids Decreases Cellular Genotoxicity and Improves Biochemical Parameters of Lipid and Glucose Metabolism. Molecules. 2018 Sep 1;23(9). pii: E2220. doi: 10.3390/molecules23092220.

9. Kim Y, Keogh JB, Clifton PM. Polyphenols and Glycemic Control. Nutrients. 2016 Jan 5;8(1). pii: E17. doi: 10.3390/nu8010017.

10. Campos VC \& Tappy L (2016) Physiological handling of dietary fructose-containing sugars: implications for health. Int J Obes (Lond) 40 Suppl 1, S6-S11.

11. Targher G, Marchesini G \& Byrne CD (2016) Risk of type 2 diabetes in patients with non-alcoholic fatty liver disease: Causal association or epiphenomenon? Diabetes Metab 42, 142-156.

12. Bahrami M, Ataie-Jafari A, Hosseini S, et al. (2009) Effects of natural honey consumption in diabetic patients: an 8-week randomized clinical trial. Int J Food Sci Nutr 60, 618-626.

13. American Diabetes Association (2016) Classification and Diagnosis of Diabetes. Diabetes Care 39 Suppl 1, S13-22.

14. Widdowson EM \& McCance RA (1935) The available carbohydrate of fruits: Determination of glucose, fructose, sucrose and starch. Biochem J 29, 151-156.

15. Dietary Guidelines Advisory Committee (2015) Scientific Report of the 2015 Dietary Guidelines Advisory Committee. Available online at: http://www.health.gov/dietaryguidelines/2015-scientificreport/

16. World Health Organization (2015) Guideline: sugars intake for adults and children. Geneva (Switzerland): World Health Organization. 
17. Franz MJ, Powers MA, Leontos C, et al. (2010) The evidence for medical nutrition therapy for type 1 and type 2 diabetes in adults. J Am Diet Assoc 110, 1852-1889.

18. Satoh K (1978) Serum lipid peroxide in cerebrovascular disorders determined by a new colorimetric method. Clin Chim Acta 90, 37-43.

19. Benzie IF \& Strain JJ (1999) Ferric reducing/antioxidant power assay: direct measure of total antioxidant activity of biological fluids and modified version for simultaneous measurement of total antioxidant power and ascorbic acid concentration. Methods Enzymol 299, 15-27.

20. Kurtze N, Rangul V \& Hustvedt BE (2008) Reliability and validity of the international physical activity questionnaire in the Nord-Trøndelag health study (HUNT) population of men. BMC Med Res Methodol 8, 63.

21. Abdulrhman M, El-Hefnawy M, Hussein R, et al. (2011) The glycemic and peak incremental indices of honey, sucrose and glucose in patients with type 1 diabetes mellitus: effects on C-peptide levela pilot study. Acta Diabeto/ 48, 89-94.

22. Raatz SK, Johnson LK \& Picklo MJ (2015) Consumption of honey, sucrose, and high-fructose corn syrup produces similar metabolic effects in glucose-tolerant and -intolerant individuals. $J$ Nutr $145,2265-2272$.

23. Akhlaghi M (2016) Non-alcoholic Fatty Liver Disease: Beneficial Effects of Flavonoids. Phytother ResPublished online: 16 Jun 2016. doi: 10.1002/ptr.5667.

24. Wabaidur SM, Ahmed YB, Alothman ZA, et al. (2015) Ultra high performance liquid chromatography with mass spectrometry method for the simultaneous determination of phenolic constituents in honey from various floral sources using multiwalled carbon nanotubes as extraction sorbents. $J$ Sep Sci 38, 2597-2606.

25. Gheldof N \& Engeseth NJ (2002) Antioxidant capacity of honeys from various floral sources based on the determination of oxygen radical absorbance capacity and inhibition of in vitro lipoprotein oxidation in human serum samples. J Agric Food Chem 50, 3050-3045.

26. Aeberli I, Hochuli M, Gerber PA, et al. (2013) Moderate amounts of fructose consumption impair insulin sensitivity in healthy young men: a randomized controlled trial. Diabetes Care 36, 150-156.

27. Stanhope KL, Bremer AA, Medici V, et al. (2011) Consumption of fructose and high fructose corn syrup increase postprandial triglycerides, LDL-cholesterol, and apolipoprotein-B in young men and women. J Clin Endocrinol Metab 96, E1596-1605.

28. Stanhope KL \& Havel PJ (2008) Fructose consumption: potential mechanisms for its effects to increase visceral adiposity and induce dyslipidemia and insulin resistance. Curr Opin Lipido/ 19, 1624.

29. Koba S, Hirano T, Murayama S, et al. (2003) Small dense LDL phenotype is associated with postprandial increases of large VLDL andremnant-like particles in patients with acute myocardial infarction. Atherosclerosis 170, 131-140.

30. Tobin KA, Ulven SM, Schuster GU, et al. (2002) Liver X receptors as insulin-mediating factors in fatty acid and cholesterol biosynthesis. J Biol Chem 277, 10691-10697. 
31. Silbernagel G, Lütjohann D, Machann J, et al. (2012) Cholesterol synthesis is associated with hepatic lipid content and dependent on fructose/glucose intake in healthy humans. Exp Diabetes Res 2012, 361863.

32. Stanhope KL, Schwarz JM, Keim NL, et al. (2009) Consuming fructose-sweetened, not glucosesweetened, beverages increases visceral adiposity and lipids and decreases insulin sensitivity in overweight/obese humans. J Clin Invest 119, 1322-1334.

33. von Frankenberg AD, Silva FM, de Almeida JC, et al. (2014) Effect of dietary lipids on circulating adiponectin: a systematic review with meta-analysis of randomised controlled trials. $\mathrm{Br} \mathrm{J}$ Nutr 112, 1235-1250.

34. Nemoseck TM, Carmody EG, Furchner-Evanson A, et al (2011) Honey promotes lower weight gain, adiposity, and triglycerides than sucrose in rats. Nutr Res 31, 55-60.

35. Swarbrick MM \& Havel PJ (2008) Physiological, pharmacological, and nutritional regulation of circulating adiponectin concentrations in humans. Metab Syndr Relat Disord 6, 87-102.

36. Rezvani R, Cianflone K, McGahan JP, et al (2013) Effects of sugar-sweetened beverages on plasma acylation stimulating protein, leptin and adiponectin: relationships with metabolic outcomes. Obesity (Silver Spring) 21, 2471-2480.

37. Pollock NK, Bundy V, Kanto W, et al. (2012) Greater fructose consumption is associated with cardiometabolic risk markers and visceral adiposity in adolescents. J Nutr 142, 251-257.

38. Mager DR, Patterson C, So S, et al. (2010) Dietary and physical activity patterns in children with fatty liver. Eur J Clin Nutr 64, 628-635.

39. Al-Waili NS (2004) Natural honey lowers plasma glucose, C-reactive protein, homocysteine, and blood lipids in healthy, diabetic, and hyperlipidemic subjects: comparison with dextrose and sucrose. J Med Food 7, 100-107.

40. Yaghoobi N, Al-Waili N, Ghayour-Mobarhan M, et al (2008) Natural honey and cardiovascular risk factors; effects on blood glucose, cholesterol, triacylglycerole, CRP, and body weight compared with sucrose. ScientificWorldJournal 8, 463-469.

\section{Tables}

Table 1 - Baseline characteristics of and medicinal use of the study subjects $(n=42)^{1}$ 


\begin{tabular}{lc}
\hline Characteristics & Mean \pm SD or n (\%) \\
\hline Age (y) & $57.5 \pm 9.8$ \\
Males (n) & $78(42.9)$ \\
\hline Duration of diabetes (y) & $160.8 \pm 9.1$ \\
\hline Height (cm) & $72.0 \pm 11.9$ \\
\hline Weight (kg) & $27.8 \pm 3.6$ \\
\hline BMI $\left(\mathrm{kg} / \mathrm{m}^{2}\right)$ & \\
\hline Hypoglycemic drugs & $40(95.2)$ \\
$\quad$ Metformin & $18(42.8)$ \\
Glibenclamide & $11(26.2)$ \\
$\quad$ Acarbose & $4(9.5)$ \\
Glitazone & $3(7.1)$ \\
Gliclazide & $1(2.4)$ \\
Repaglinide & \\
\hline Lipid-lowering drugs & $42(100)$ \\
Atorvastatin & $2(4.8)$ \\
Gemfibrozil &
\end{tabular}

BMI, body mass index.

Table 2 - The effect of 8 weeks honey consumption on main study outcomes (values are related to the both sequences)! 


\begin{tabular}{|c|c|c|c|c|c|c|}
\hline & Baseline & Week 8 & $\begin{array}{l}\text { Difference } \\
(95 \% \mathrm{CI})\end{array}$ & $\begin{array}{l}\mathrm{p} \text { value } \\
{\text { (time })^{2}}^{2}\end{array}$ & $\begin{array}{c}\mathrm{p} \text { value } \\
\text { (treatment } \times \\
\text { time) }{ }^{3} \\
\end{array}$ & $\begin{array}{l}\mathrm{p} \text { value (carryover } \\
\text { effect) }^{4}\end{array}$ \\
\hline \multicolumn{7}{|l|}{$\begin{array}{l}\text { Fasting blood glucose } \\
(\mathrm{mg} / \mathrm{dl})\end{array}$} \\
\hline Control condition & $\begin{array}{c}129.1 \pm \\
36.8\end{array}$ & $\begin{array}{c}133.7 \pm \\
33.9\end{array}$ & $\begin{array}{c}4.67(-5.79 \text { to } \\
15.1)\end{array}$ & 0.4 & 0.8 & 0.7 \\
\hline Honey condition & $\begin{array}{c}136.1 \pm \\
45.3\end{array}$ & $\begin{array}{c}138.9 \pm \\
38.8\end{array}$ & $\begin{array}{c}2.83(-10.7 \text { to } \\
16.4)\end{array}$ & 0.7 & & \\
\hline \multicolumn{7}{|l|}{ Triglycerides (mg/dl) } \\
\hline Control condition & $\begin{array}{c}124.1 \pm \\
58.4\end{array}$ & $\begin{array}{c}143.3 \pm \\
92.6\end{array}$ & $\begin{array}{c}19.2(-4.7 \text { to } \\
43.1)\end{array}$ & 0.1 & 0.4 & 0.1 \\
\hline Honey condition & $\begin{array}{c}126.2 \pm \\
55.6\end{array}$ & $\begin{array}{c}134.1 \pm \\
73.3\end{array}$ & $\begin{array}{c}7.9(-6.3 \text { to } \\
22.1)\end{array}$ & 0.3 & & \\
\hline
\end{tabular}

Total cholesterol

(mg/dl)

\begin{tabular}{|ccccccc} 
Control condition & $158.2 \pm$ & $152.7 \pm$ & $-5.5(-14.8$ to & 0.2 & 0.4 & 0.8 \\
& 41.6 & 37.2 & $3.8)$ & & \\
\hline Honey condition & $158.8 \pm$ & $157.3 \pm$ & $-1.5(-8.6$ to 5.7$)$ & 0.7 & & \\
& 43.3 & 33.4 & & &
\end{tabular}

LDL cholesterol (mg/dl)

$\begin{array}{lcccccc}\text { Control condition } & 96.4 \pm & 80.2 \pm & -16.2(-23.5 \text { to } & <0.001 & 0.005 & 0.7 \\ & 32.3 & 27.7 & -8.9) & & \\ \text { Honey condition } & 90.3 \pm & 88.6 \pm & -1.7(-8.2 \text { to } 4.9) & 0.6 & & \end{array}$

HDL cholesterol (mg/dl)

\begin{tabular}{lccccc} 
Control condition & $38.7 \pm 8.3$ & $47.9 \pm$ & $9.2(5.6$ to 12.9$)$ & $<0.001$ & 1 \\
& \multicolumn{5}{c}{14.6} \\
Honey condition & $39.2 \pm$ & $48.3 \pm$ & $9.1(5.7$ to 12.5$)$ & $<0.001$ \\
& 10.6 & 11.9 &
\end{tabular}

Malondialdehyde

( $\mu \mathrm{mol} / \mathrm{l}$ )

\begin{tabular}{|c|c|c|c|c|c|}
\hline Control condition & $3.3 \pm 0.953 .1 \pm 0.80$ & $\begin{array}{c}-0.19(-0.48 \text { to } \\
0.10)\end{array}$ & 0.2 & 0.7 & 0.09 \\
\hline Honey condition & $3.5 \pm 0.863 .1 \pm 0.70$ & $\begin{array}{c}-0.38(-0.67 \text { to } \\
-0.09)\end{array}$ & 0.01 & & \\
\hline
\end{tabular}

Total antioxidant capacity (mmol/L)

$\begin{array}{lcccccc}\text { Control condition } & 0.85 \pm & 0.70 \pm & -0.15(-0.32 \text { to } & 0.07 & 0.8 & 0.08 \\ & 0.38 & 0.33 & 0.01) & & \\ \text { Honey condition } & 0.73 \pm & 0.67 \pm & -0.06(-0.22 \text { to } & 0.4 & \\ & 0.42 & 0.36 & 0.09) & & \end{array}$

hs-CRP (mg/L)

\begin{tabular}{lcccccc} 
Control condition & $0.62 \pm$ & $0.58 \pm$ & $-0.04(-0.09$ to & 0.2 & 0.1 & 0.1 \\
& 0.18 & 0.18 & $0.02)$ & & \\
Honey condition & $0.66 \pm$ & $0.59 \pm$ & $-0.07(-0.11$ to & 0.003 & \\
& 0.14 & 0.11 & $-0.02)$ & & \\
& \multicolumn{5}{c}{ Page $14 / 16$} &
\end{tabular}


Adiponectin (mg/L)

\begin{tabular}{lcccccc} 
Control condition & $9.9 \pm 2.5$ & $9.8 \pm 2.4$ & $\begin{array}{c}-0.07(-0.73 \text { to } \\
0.60)\end{array}$ & 0.8 & 0.005 & 0.02 \\
\hline Honey condition & $10.5 \pm 1.6$ & $9.7 \pm 1.7$ & $\begin{array}{c}-0.75(-1.1 \text { to } \\
0.38)\end{array}$ & $<0.001$ &
\end{tabular}

${ }^{1}$ Data are means \pm SD $(n=42) .{ }^{2}$ Comparisons between pre- and post- values of each condition was done by paired samples' $t$ test. ${ }^{3}$ Between-group comparisons were performed by ANCOVA with adjustments for the corresponding basal values. ${ }^{4}$ Carryover effect was estimated by ANOVA test for a $2 \times 2$ crossover study. Abbreviations: CI, confidence interval; HDL, high-density lipoprotein; hs-CRP, high sensitivity-C reactive protein; LDL, low-density lipoprotein.

Table 3 - Dietary intakes and physical activity of participants during the study (values are related to the both sequences) $)^{1}$

\begin{tabular}{|c|c|c|c|c|c|c|c|c|c|}
\hline & \multicolumn{3}{|c|}{ Control condition } & \multirow[b]{2}{*}{$\begin{array}{c}\mathrm{p} \\
\text { value }^{2}\end{array}$} & \multicolumn{3}{|c|}{ Honey condition } & \multirow[b]{2}{*}{$\begin{array}{c}\mathbf{p} \\
\text { value }^{2}\end{array}$} & \multirow[b]{2}{*}{$\begin{array}{c}p \\
\text { value }^{3}\end{array}$} \\
\hline & Baseline & Week 8 & $\begin{array}{c}\text { Difference } \\
(95 \% \mathrm{CI})\end{array}$ & & Baseline & Week 8 & $\begin{array}{c}\text { Difference } \\
(95 \% \mathrm{CI})\end{array}$ & & \\
\hline Energy (kcal/day) & $\begin{array}{c}1516 \pm \\
664\end{array}$ & $\begin{array}{c}1472 \pm \\
500\end{array}$ & $\begin{array}{c}-44.7 \\
(-222 \text { to } \\
132)\end{array}$ & 0.6 & $\begin{array}{c}1423 \pm \\
479\end{array}$ & $\begin{array}{c}1557 \pm \\
460\end{array}$ & $\begin{array}{c}134 \\
(264 \text { to } \\
324)\end{array}$ & 0.045 & 0.1 \\
\hline $\begin{array}{l}\text { Carbohydrates (\% of } \\
\text { energy) }\end{array}$ & $61.9 \pm 7.4$ & $60.8 \pm 8.1$ & $\begin{array}{c}-1.1 \\
(-3.8 \text { to } \\
1.6)\end{array}$ & 0.4 & $64.3 \pm 8.7$ & $66.2 \pm 7.3$ & $\begin{array}{c}1.9 \\
(-0.79 \text { to } \\
4.5)\end{array}$ & 0.2 & 0.1 \\
\hline Fat (\% of energy) & $26.3 \pm 8.2$ & $27.6 \pm 7.9$ & $\begin{array}{c}1.3 \\
(-0.82 \text { to } \\
3.4)\end{array}$ & 0.2 & $24.8 \pm 7.3$ & $23.4 \pm 6.6$ & $\begin{array}{c}-1.3 \\
(-3.2 \text { to } \\
0.54)\end{array}$ & 0.2 & 0.06 \\
\hline $\begin{array}{l}\text { Saturated fats (\% of } \\
\text { energy) }\end{array}$ & $7.0 \pm 3.2$ & $7.6 \pm 2.8$ & $\begin{array}{c}0.69 \\
(-0.24 \text { to } \\
1.6)\end{array}$ & 0.1 & $7.5 \pm 3.6$ & $6.8 \pm 2.9$ & $\begin{array}{c}-0.66 \\
(-2.0 \text { to } \\
0.72)\end{array}$ & 0.3 & 0.1 \\
\hline Cholesterol (g/day) & $\begin{array}{c}153.5 \pm \\
144.6\end{array}$ & $\begin{array}{c}176.9 \pm \\
128.4\end{array}$ & $\begin{array}{c}23.4 \\
(-22.4 \text { to } \\
69.3)\end{array}$ & 0.3 & $\begin{array}{c}114.0 \pm \\
10.8\end{array}$ & $\begin{array}{c}143.5 \pm \\
112\end{array}$ & $\begin{array}{c}29.4 \\
(-12.1 \text { to } \\
71.0)\end{array}$ & 0.2 & 0.8 \\
\hline Sucrose (\% of energy) & $2.2 \pm 2.2$ & $2.8 \pm 2.8$ & $\begin{array}{c}0.62 \\
(-0.36 \text { to } \\
1.6)\end{array}$ & 0.2 & $1.9 \pm 2.0$ & $2.4 \pm 2.3$ & $\begin{array}{c}0.54 \\
(-0.43 \text { to } \\
1.5)\end{array}$ & 0.3 & 0.9 \\
\hline $\begin{array}{l}\text { Fructose (\% of } \\
\text { energy) }\end{array}$ & $1.8 \pm 1.3$ & $2.5 \pm 2.7$ & $\begin{array}{c}0.71 \\
(-0.08 \text { to } \\
1.5)\end{array}$ & 0.08 & $2.2 \pm 2.5$ & $7.6 \pm 2.0$ & $\begin{array}{c}5.4 \\
(4.5 \text { to } 6.3)\end{array}$ & $<0.001$ & $<0.001$ \\
\hline Fiber (g/day) & $12.1 \pm 5.2$ & $12.9 \pm 5.4$ & $\begin{array}{c}0.81 \\
(-0.98 \text { to } \\
2.6)\end{array}$ & 0.4 & $13.7 \pm 5.5$ & $11.8 \pm 5.1$ & $\begin{array}{c}-2.0 \\
(-4.0 \text { to } \\
0.09)\end{array}$ & 0.06 & 0.04 \\
\hline $\begin{array}{l}\text { Physical activity } \\
\text { (min/day) }\end{array}$ & $\begin{array}{c}180.0 \pm \\
245.4\end{array}$ & $\begin{array}{c}148.2 \pm \\
177.3\end{array}$ & $\begin{array}{c}-31.8 \\
(-96.5 \text { to } \\
32.8)\end{array}$ & 0.3 & $\begin{array}{c}154.7 \pm \\
141.9\end{array}$ & $\begin{array}{c}141.9 \pm \\
169.6\end{array}$ & $\begin{array}{c}-12.8 \\
(-59.1 \text { to } \\
33.5)\end{array}$ & 0.6 & 0.7 \\
\hline
\end{tabular}


${ }^{1}$ Data are means \pm SD $(n=42) .{ }^{2}$ p value assessed by paired samples $t$ test. ${ }^{3}$ Between-group comparisons of dietary intakes were tested with independent t-test.

\section{Figures}



Figure 1

CONSORT flow chart.

\section{Supplementary Files}

This is a list of supplementary files associated with this preprint. Click to download.

- supplement1.doc 